\title{
Impurity and vacancy effects in graphene
}

\author{
V.M. Loktev \\ Bogolyubov Institute for Theoretical Physics, NAS of Ukraine, 14 b Metrolohichna Str., Kiev 03680, Ukraine \\ E-mail: vloktev@bitp.kiev.ua \\ Yu.G. Pogorelov \\ IFIMUP/Departamento de Fisica, Universidade do Porto, R. Campo Alegre, 687, Porto, 4169-007, Portugal \\ E-mail: ypogorel@fc.up.pt
}

Received May 15, 2012

\begin{abstract}
A Green function analysis has been developed for quasiparticle spectrum of a 2D graphene sheet in presence of different types of substitutional disorder, including vacancies. The anomalous character of impurity effects in this system is demonstrated, compared to those in well known doped semiconductors, and explained in terms of conical singularities in the band spectrum of pure graphene. The criteria for appearance of localized states on clusters of impurity scatterers and for qualitative restructuring of band spectrum are established and a possibility for a specific metal/insulator transition at presence of vacancies is indicated.
\end{abstract}

PACS: 03.65.Pm Relativistic wave equations;

71.30.+h Metal-insulator transitions and other electronic transitions;

71.55.-i Impurity and defect levels.

Keywords: graphene, quasiparticle spectrum, impurity and vacancy effects.

\section{Introduction}

There is a growing attention to electronic properties of a single carbon layer known as graphene [1]. Its $2 \mathrm{D}$ honeycomb lattice defines a peculiar band structure [2] with two nodal points in the Brillouin zone (BZ) where conical energy surfaces (with zero effective mass) of conduction and valence bands touch each other. This gives rise to electronic dynamics of relativistic Dirac type [3], extraordinary for condensed matter, and generates such unusual phenomena as half-integer Hall effect [4-6] and, possibly, the magnetic catalysis of an excitonic gap [7,8], ferromagnetism and superconductivity [9]. On the other hand, it is of interest to examine the effects that various kinds of impurities can produce on this remarkable material, regarding for instance a fundamental role of such effects in physics of common semiconductors (with finite effective mass) [10]. An intriguing situation with impurity levels near conical singularities was recognized in $d$-wave superconductors [11] where theoretical predictions are sometimes contradictory [12] and not fully confirmed by the existing experimental data. To this time, the disorder effects in graphene were theoretically studied, searching for weak localization in this $2 \mathrm{D}$ electronic system under weak scattering (Born limit) $[13,14]$ or for strong localization under infinitely strong (unitary limit) perturbation [15]. This work is aimed on a consequent description of restructured electronic spectrum, at arbitrary perturbation strength and in a rather broad range of impurity concentration, and on specifics of this restructuring for Dirac quasiparticles under realistic perturbation, compared to more common quasiparticles with parabolic dispersion. In this respect, our results contribute to the overall topic of the present issue dedicated to Prof. E.V. Savchenko's jubilee and her notable works on the electronically induced defects, especially vacancies, in cryocrystals [16-18].

\section{Hamiltonian and Green functions}

Let us start from the simplest tight-binding Hamiltonian restricted to nearest neighbor hopping

$$
H=t \sum_{\mathbf{n}, \boldsymbol{\delta}} a_{\mathbf{n}}^{\dagger} b_{\mathbf{n}+\boldsymbol{\delta}}+\text { h.c., }
$$

where $t$ is the hopping amplitude, $a_{\mathbf{n}}$ and $b_{\mathbf{n}+\boldsymbol{\delta}}$ are the Fermi operators of (spinless) electrons on sites of type 1 and 2, respectively, and the atomic energy on each site is chosen as zero energy reference. The set of vectors $\boldsymbol{\delta}$ (of length $|\boldsymbol{\delta}|=a$ ) relates to three nearest neighbors of a type 1 site (due to lack of central symmetry, it does not coincide with the set of $-\boldsymbol{\delta}$ for nearest neighbors of a type 2 
site, see Fig. 1,a). Passing from site operators to plane waves: $a_{\mathbf{k}}=N^{-1 / 2} \sum_{\mathbf{n}} \mathrm{e}^{-i \mathbf{k n}} a_{\mathbf{n}}$ and $b_{\mathbf{k}}=N^{-1 / 2} \sum_{\mathbf{n}} \mathrm{e}^{-i \mathbf{k n}} b_{\mathbf{n}}$ ( $N$ being the number of cells in the lattice) the Hamiltonian, Eq. (1), is splitted in quasimomentum $\mathbf{k}$ :

$$
H=t \sum_{\mathbf{k}}\left(\gamma_{\mathbf{k}} a_{\mathbf{k}}^{\dagger} b_{\mathbf{k}}+\text { h.c. }\right)
$$

with the complex function

$$
\gamma_{\mathbf{k}}=\sum_{\boldsymbol{\delta}} \mathrm{e}^{i \mathbf{k} \cdot \boldsymbol{\delta}}=\mathrm{e}^{-i a k_{x}}+2 \mathrm{e}^{i a k_{x} / 2} \cos \frac{\sqrt{3} a k_{y}}{2} .
$$

It is then fully diagonalized:

$$
H=\sum_{\mathbf{k}} \varepsilon_{\mathbf{k}}\left(\alpha_{\mathbf{k}}^{\dagger} \alpha_{\mathbf{k}}-\beta_{\mathbf{k}}^{\dagger} \beta_{\mathbf{k}}\right)
$$

in terms of the normal modes operators:

$$
\begin{aligned}
& \alpha_{\mathbf{k}}=\frac{1}{\sqrt{2}}\left(\mathrm{e}^{-i \varphi_{\mathbf{k}} / 2} a_{\mathbf{k}}+\mathrm{e}^{i \varphi_{\mathbf{k}} / 2} b_{\mathbf{k}}\right), \\
& \beta_{\mathbf{k}}=\frac{1}{\sqrt{2}}\left(\mathrm{e}^{i \varphi_{\mathbf{k}} / 2} b_{\mathbf{k}}-\mathrm{e}^{-i \varphi_{\mathbf{k}} / 2} a_{\mathbf{k}}\right),
\end{aligned}
$$
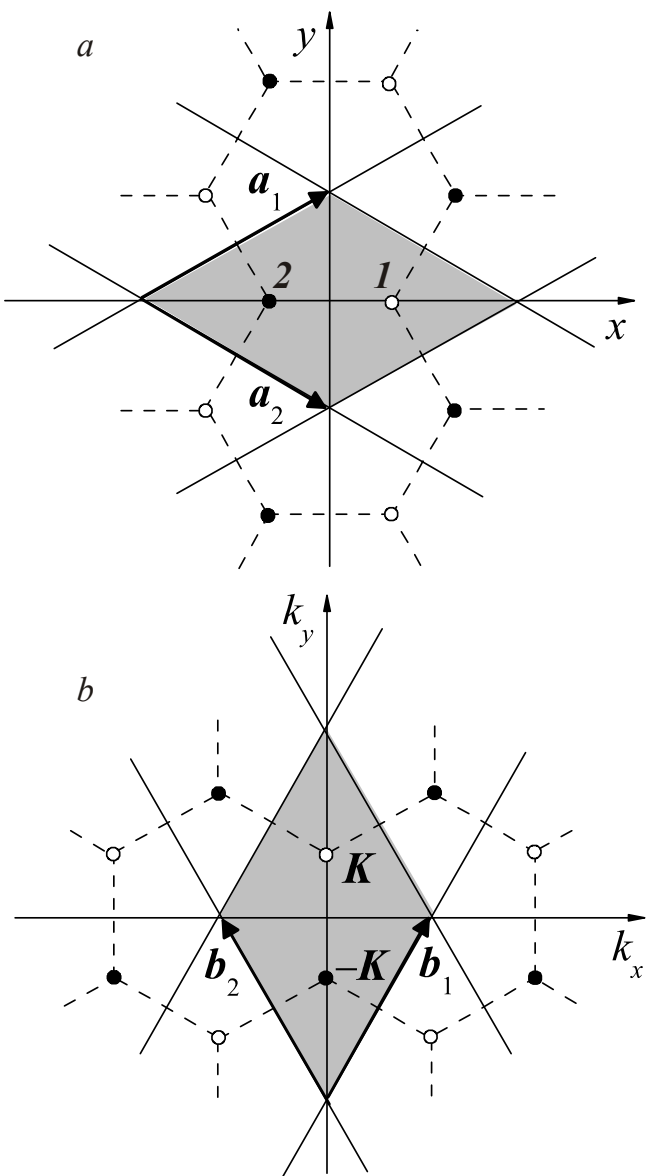

Fig. 1. 2D lattice structure of a graphene sheet. Rhombic primitive cell (shadowed) with two non-equivalent positions for carbon atoms, 1 (open circles) and 2 (solid circles), and elementary translation vectors $a_{1}$ and $a_{2}$ of length $a \sqrt{3}(a)$. Rhombic Brillouin zone (shadowed) with two non-equivalent nodal points, $\mathbf{K}$ (open) and $-\mathbf{K}$ (solid), and vectors of reciprocal lattice $\mathbf{b}_{1}$ and $\mathbf{b}_{2}$ of length $4 \pi /(a \sqrt{3})(b)$. with the eigen-energies $\varepsilon_{\mathbf{k}}=t\left|\gamma_{\mathbf{k}}\right|$. The main specifics of graphene is that the function $\gamma_{\mathbf{k}}$ vanishes near two isolated points in the BZ: $\mathbf{K}=(0,4 \pi / 3 \sqrt{3} a)$ and $-\mathbf{K}$ (Fig. 1). Also the lack of central symmetry for the point group $C_{3}$ makes this vanishing linear in a small difference $\mathbf{q}=\mathbf{k}-\mathbf{K}($ or $\mathbf{q}=\mathbf{k}+\mathbf{K})$ :

$$
\gamma_{\mathbf{k}} \equiv \gamma_{\mathbf{q}} \approx-\frac{3}{2} a\left(q_{y}+i q_{x}\right), \text { at } a q \ll 1,
$$

defining the conical form of isoenergetic surfaces $\varepsilon= \pm \varepsilon_{\mathbf{q}}$ as

$$
\varepsilon_{q} \approx \hbar v_{F} q
$$

with the Fermi velocity $v_{F}=\frac{3}{2} t a / \hbar$. This conical spectrum is commonly compared with the relativistic massless Dirac fermions. The following analysis of this system is restricted to the low energy physics, essentially determined by the vicinities (below also referred to as valleys) of two nodal points $\pm \mathbf{K}$.

The electronic quasiparticle states at given $\mathbf{k}$ can be suitably generated by the operator spinors:

$$
\psi_{\mathbf{k}}=\left(\begin{array}{c}
a_{\mathbf{k}} \\
b_{\mathbf{k}}
\end{array}\right)
$$

and the Hamiltonian, Eq. (1), is expressed through such spinors as:

$$
H=\sum_{\mathbf{k}} \varepsilon_{\mathbf{k}} \psi_{\mathbf{k}}^{\dagger} \hat{h}_{\mathbf{k}} \psi_{\mathbf{k}}
$$

with the matrix $\hat{h}_{\mathbf{k}}=t\left(\gamma_{\mathbf{k}} \hat{\tau}_{+}+\gamma_{\mathbf{k}}^{*} \hat{\tau}_{-}\right)$, and $\hat{\tau}_{ \pm}=\hat{\tau}_{x} \pm i \hat{\tau}_{y}$ involving Pauli matrices $\hat{\tau}_{i}$. In what follows, the integration in $\mathbf{k}$ over BZ is presented as twice of that in $\mathbf{q}$, done in an approximate way over a circle of radius $q_{m}=\sqrt{8 \pi / 3 \sqrt{3}} / a$ around $\mathbf{K}$ (half of the full BZ area). The corresponding effective bandwidth $W=\hbar v_{F} q_{m} \approx 2.87 t$, by the approximate dispersion law $\varepsilon_{\mathbf{q}}$, is close to the exact bandwidth $3 t$, by the exact dispersion law $\varepsilon_{\mathbf{k}}$.

We describe the dynamics of this system by the (Fourier transformed) two-time Green functions (GF's) [19], here combined into a $2 \times 2$ matrix $\hat{G}_{\mathbf{k}, \mathbf{k}^{\prime}}=\left\langle\left\langle\psi_{\mathbf{k}} \mid \psi_{\mathbf{k}^{\prime}}^{\dagger}\right\rangle\right\rangle$ and satisfying the equation of motion:

$$
\varepsilon\left\langle\left\langle\psi_{\mathbf{k}} \mid \psi_{\mathbf{k}^{\prime}}^{\dagger}\right\rangle\right\rangle=\delta_{\mathbf{k}, \mathbf{k}^{\prime}}+\left\langle\left\langle\left[\psi_{\mathbf{k}}, H\right] \mid \psi_{\mathbf{k}^{\prime}}^{\dagger}\right\rangle\right\rangle
$$

where the commutator $\left[\psi_{\mathbf{k}}, H\right]$ is understood as a spinor whose components are commutators of respective $\psi_{\mathbf{k}}$ components with $H:\left[\psi_{\mathbf{k}}, H\right]_{j}=\left[\psi_{\mathbf{k}, j}, H\right]$. For the unperturbed system with the Hamiltonian, Eq. (5), the exact form of GF is readily obtained from Eq. (6) as $\hat{G}_{\mathbf{k}, \mathbf{k}^{\prime}}=\hat{g}_{\mathbf{k}} \delta_{\mathbf{k}, \mathbf{k}^{\prime}}$ where

$$
\hat{g}_{\mathbf{k}}=\frac{\varepsilon+\hat{h}_{\mathbf{k}}}{\varepsilon^{2}-\varepsilon_{\mathbf{k}}^{2}} .
$$

The observable characteristics of the system follow from the Green functions in a usual way, for instance, the density of states (DOS) is obtained as

$$
\rho(\varepsilon)=\operatorname{Im} \operatorname{Tr} \frac{1}{\pi N} \sum_{\mathbf{k}} \hat{G}_{\mathbf{k}, \mathbf{k}} .
$$


Another important quantity, the local density of states (LDOS) on a site $\mathbf{n}_{j}$ of $j$ th type:

$$
\rho_{\mathbf{n}_{j}}(\varepsilon)=\frac{1}{\pi N} \operatorname{Im} \sum_{\mathbf{k}, \mathbf{k}^{\prime}} \mathrm{e}^{i\left(\mathbf{k}-\mathbf{k}^{\prime}\right) \mathbf{n}_{j}} \hat{G}_{\mathbf{k}, \mathbf{k}^{\prime}} \hat{\tau}_{j},
$$

with the projecting matrices $\hat{\tau}_{j}=\left[1+(-1)^{j} \hat{\tau}_{z}\right] / 2$. In the unperturbed system, the exact solution, Eq. (7), leads to simple linear energy dependencies of DOS: $\rho^{0}(\varepsilon)=\varepsilon / W^{2}$, and also of LDOS: $\rho_{\mathbf{n}_{j}}^{0}(\varepsilon)=\rho^{0}(\varepsilon) / 2$. The following analysis is focused on modifications of these dependencies under local perturbations by impurities or vacancies.

\section{Impurity perturbations}

The notable distinction of graphene from genuine relativistic systems of quantum field theory is the possibility to study the effects of localized perturbations on its dynamics, which is our main purpose here. The disordered systems are specific by presence of localized states whose wave functions are restricted to atomically short distances $[20,21]$, they always appear near the limits of nonperturbed energy spectrum but can either emerge near some impurity resonance energies [22]. Besides them, extended (also called Bloch-like) states are present, similar to common Bloch waves in perfect crystals but with limitation of wave function coherence by certain (long enough) mean free path $\ell$.

Here we adopt the Lifshitz model of impurity perturbation, where the perturbation potential is identical for all impurity sites randomly distributed among the lattice sites [20]. This model looks more adequate to the case of rare defects in graphene, than the alternative choice [23] of Anderson model with random perturbations at each lattice cite [24]. Within the tight-binding framework, two characteristic types of local perturbation are considered: i) that on the on-site atomic energy, also referred to as the "diagonal disorder" [25], here more relevant for substitution impurities and ii) that on the inter-site hopping, or the "nondiagonal disorder", more relevant for vacancies.

We begin from the simplest point-like diagonal perturbation by a Lifshitz impurity, producing a certain shift $V$ of the on-site energy at the impurity site. We denote $p_{1}$ the defect sites of type 1 with concentration $c_{1}$ and $p_{2}$ those of type 2 with concentration $c_{2}$ (not necessarily equal to $c_{1}$ ), the total impurity concentration being $c_{1}+c_{2}=c \ll 1$. Then the perturbation operator in terms of local Fermi operators appears as

$$
H^{\prime}=V\left(\sum_{\mathbf{p}_{1}} a_{\mathbf{p}_{1}}^{\dagger} a_{\mathbf{p}_{1}}+\sum_{\mathbf{p}_{2}} b_{\mathbf{p}_{2}}^{\dagger} b_{\mathbf{p}_{2}}\right),
$$

and, passing to the spinor representation, Eq. (5), it gets the form of scattering operator:

$$
H^{\prime}=\frac{V}{N} \sum_{\mathbf{k}, \mathbf{k}^{\prime \prime}} \psi_{\mathbf{k}}^{\dagger} \sum_{j, \mathbf{p}_{j}} \mathrm{e}^{i\left(\mathbf{k}^{\prime}-\mathbf{k}\right) \mathbf{p}_{j}} \hat{\tau}_{j} \psi_{\mathbf{k}^{\prime}}
$$

The equation of motion for the perturbed GF matrix, Eq. (7) with Hamiltonian $H+H^{\prime}$, takes the form:

$$
\hat{G}_{\mathbf{k}, \mathbf{k}^{\prime}}=\hat{g}_{\mathbf{k}}\left[\delta_{\mathbf{k}, \mathbf{k}^{\prime}}+\frac{V}{N} \sum_{\mathbf{k}^{\prime \prime}, \sum_{j}, \hat{\mathbf{p}}_{j}} \mathrm{e}^{i\left(\mathbf{k}^{\prime \prime}-\mathbf{k}\right) \mathbf{p}_{j}} \hat{G}_{\mathbf{k}^{\prime \prime}, \mathbf{k}^{\prime}}\right]
$$

the sum in the brackets presenting a set of "scattered" GF's by impurity perturbations. Then certain iteration routines on the scattered GF's $\hat{G}_{\mathbf{k}^{\prime \prime}, \mathbf{k}^{\prime}}$ lead to solutions for the initial (or "master") GF as certain expansions in groups of impurity scatterers, called group expansions (GE's), specific for band-like or localized states in the disordered system [22].

Namely, the band-like states are better described by the so-called fully renormalized GE, resulting from consecutive iterations of Eq. (12) on each scattered GF except those already present in the previous iterations. Collecting the same GF's from all the iterations forms some coefficients beside them as infinite series of respective scattering amplitudes by all multiple scatterings. The subsequent algebraic solution of resulting chained equations either for the master GF and its posterior scattered ones can be closed so that the master GF gets expanded in terms of multiple scatterings on a single impurity, on a pair of impurities, etc. The full renormalization means that each element of such expansion also presents as a similar expansion (but subdued to a particular scattering history of the master GF), and so on for its own elements. Such hierarchical structure is assured by the fact that one can uniquely trace the scattering history for each element at each level of the expansion. The most important momentum-diagonal $\mathrm{GF}$ is written as:

$$
\hat{G}_{\mathbf{k}} \equiv \hat{G}_{\mathbf{k}, \mathbf{k}}=\left(\hat{g}_{\mathbf{k}}^{-1}-\hat{\Sigma}\right)^{-1}
$$

that is the inverse GF involves the self-energy matrix in the GE form:

$$
\begin{aligned}
& \hat{\Sigma}=\hat{T} \sum_{j} c_{j} \hat{\tau}_{j} {\left[1+\sum_{j^{\prime}, \mathbf{n} \neq 0} c_{j^{\prime}}\left(\mathrm{e}^{-i \mathbf{k} \cdot \mathbf{n}} \hat{A}_{\mathbf{n}} \hat{\tau}_{j^{\prime}}+\hat{A}_{\mathbf{n}} \hat{\tau}_{j^{\prime}} \hat{A}_{-\mathbf{n}} \hat{\tau}_{j}\right) \times\right.} \\
&\left.\times\left(1-\hat{A}_{\mathbf{n}} \hat{\tau}_{j^{\prime}} \hat{A}_{-\mathbf{n}} \hat{\tau}_{j}\right)^{-1}+\ldots\right] .
\end{aligned}
$$

Here $\hat{T}=V(1-\hat{G} V)^{-1}$ is the common T-matrix, the projectors $\hat{\tau}_{j}$ refer to the types of impurity scatterers and the matrix of effective interaction:

$$
\hat{A}_{\mathbf{n}}=\frac{1}{N} \sum_{\mathbf{k}}^{\prime} \mathrm{e}^{i \mathbf{k} \cdot \mathbf{n}} \hat{G}_{\mathbf{k}} \hat{T} .
$$

in a sequence $\hat{\tau}_{j} \hat{A}_{\mathbf{n}} \hat{\tau}_{j^{\prime}}$ describes electron scatterings on a pair of impurities of types $j$ and $j^{\prime}$ at separation $\mathbf{n}$. 
The primed sum in Eq. (15) is subdued to the history before this scattering, that is restricted only to momenta noncoincident with its preceding ones in products like $\hat{\tau}_{j} \hat{A}_{\mathbf{n}} \hat{\tau}_{j^{\prime}} \hat{A}_{-\mathbf{n}} \ldots \hat{A}_{\mathbf{n}} \hat{\tau}_{j^{\prime}} \hat{A}_{-\mathbf{n}} \hat{\tau}_{j}$. Thus the sum next to the unity in the brackets of Eq. (14) defines the contribution to GE by impurity pairs and the dropped terms are due to impurity triples, etc. [22].

Other iteration routine, applied to all the scattered GF's with no exception to collect all the terms due to the free term $\hat{g}_{\mathbf{k}} \delta_{\mathbf{k}, \mathbf{k}^{\prime}}$ at any iteration of Eq. (13), leads to the nonrenormalized representation:

$$
\hat{G}_{\mathbf{k}}=\hat{g}_{\mathbf{k}}+\hat{g}_{\mathbf{k}} \hat{\sigma} \hat{g}_{\mathbf{k}}
$$

more adequate for localized states. The respective GE for the self-energy $\hat{\sigma}$ appears in the GF itself, instead of its inverse in Eq. (13), and has a similar structure to Eq. (14) but with the non-renormalized T-matrix $\hat{t}=V /(1-\hat{g} V)$, $\hat{g}=N^{-1} \sum_{\mathbf{k}} \hat{g}_{\mathbf{k}}$, and pair scattering matrices $\hat{a}_{\mathbf{n}}=\hat{g}_{\mathbf{n}} \hat{t}$ having no restrictions in quasimomentum sums. This representation is easier for practical evaluation of impurity effects and can also serve as an approximation for the (more involved) fully renormalized representation. Generally, each representation only makes sense until the corresponding GE is convergent that is qualitatively checked below by comparison of the contributions by T-matrix and pair terms.

In the low-energy approximation, Eq. (4), one has $\sum_{\mathbf{k}} \gamma_{\mathbf{k}} F\left(\varepsilon_{\mathbf{k}}\right) \approx 0$ for any regular function $F$ and can neglect the nondiagonal elements in the above local GF matrix $\hat{g}$ to present it as a scalar:

$$
\hat{g} \approx g(\varepsilon)=-\frac{\varepsilon}{W^{2}} \ln \left(1-\frac{W^{2}}{\varepsilon^{2}}\right) .
$$

Then the T-matrix also turns a scalar: $\hat{t} \approx V / D(\varepsilon)$, whose denominator $D(\varepsilon)=1-\operatorname{Vg}(\varepsilon)$ would define a singleimpurity resonance at the energy $\varepsilon=\varepsilon_{\text {res }}$ such that $\operatorname{Re} D\left(\varepsilon_{\text {res }}\right)=0$. But, this requires a strong enough perturbation: $|V|>V_{\text {cr, }}$, and, accordingly to Eq. (17), $V_{\text {cr }}=W \sqrt{1+\mathrm{e}^{2}} / 2 \approx 1.45 W$ amounts up to $V_{\text {cr }} \approx 10.4 \mathrm{eV}$ (with a common choice of $t \approx 2.5 \mathrm{eV}$ ). Of course, this is not an easy condition for real impurities like $\mathrm{N}$ or B substitutes for $\mathrm{C}$ in the graphene plane, as can be seen from rough estimates of related perturbation parameters, by the difference of corresponding ionization potentials: $V_{\mathrm{B}} \approx I_{\mathrm{C}}-I_{\mathrm{B}} \approx 3 \mathrm{eV}$ and $V_{\mathrm{N}} \approx I_{\mathrm{C}}-I_{\mathrm{N}} \approx-3.2 \mathrm{eV}$. Then, in a realistic situation when $|V|<V_{\text {cr }}$, impurity effects would mainly affect the conical point in the energy spectrum.

The choice between the above two GE forms for given $\mathbf{k}$ is done through the known Ioffe-Regel-Mott (IRM) criterion for band-like states $k \ell \gg 1[26,21]$, which can be written in more detail as:

$$
\mathbf{k} \cdot \nabla_{\mathbf{k}} \tilde{\varepsilon}_{\mathbf{k}} \gg \Gamma_{\mathbf{k}}
$$

in terms of the renormalized dispersion law $\tilde{\varepsilon}_{\mathbf{k}}=\varepsilon_{\mathbf{k}}-\operatorname{Re} \Sigma\left(\tilde{\varepsilon}_{\mathbf{k}}\right)$ and its broadening $\Gamma_{\mathbf{k}}=\operatorname{Im} \Sigma\left(\tilde{\varepsilon}_{\mathbf{k}}\right)$. Since $\operatorname{Re} \Sigma(\varepsilon)$ is almost constant at low energies, for $\mathbf{k} \approx \mathbf{K}$, the left hand side of Eq. (18) is close to $\hbar v_{F} K \sim W$ here, while the right hand side is much smaller, as shown below. Thus the IRM criterion, in the form accepted by Eq. (18), is granted and all the states are bandlike in this k-range.

Then, using the renormalized form, Eq. (14), approximated to its first term, we obtain the self-consistency equation:

$$
G_{s c}(\varepsilon)=g\left(\varepsilon-\Sigma_{s c}\right),
$$

with the related self-energy $\Sigma_{s c}(\varepsilon)=c V /\left[1-V G_{s c}(\varepsilon)\right]$ entering the argument of the $g$-function, Eq. (17). It admits a simple solution, revealing the most evident impurity effect as the shift of the nodal point by $c V: G_{s c}(\varepsilon) \approx g(\tilde{\varepsilon})$ for all $\tilde{\varepsilon}=\varepsilon-c V$, except for an exponentially narrow vicinity of the shifted nodal point, $|\tilde{\varepsilon}| \lesssim \delta=\left(2 c V^{2} / W\right) \exp \left(-W^{2} / 2 c V^{2}\right)$ [27], where it behaves as:

$$
G_{s c}(\varepsilon) \approx-\frac{1}{2}\left(\frac{W}{c V^{2}}\right)^{2}(\tilde{\varepsilon}-i \delta) .
$$

By this solution, DOS at the shifted nodal point would drop to an exponentially low minimum value,

$$
\rho_{s c, \min } \approx \frac{W}{\pi c V^{2}} \exp \left(-\frac{W^{2}}{2 c V^{2}}\right) .
$$

However some stronger contributions can come from the next GE terms as shown below.

Since the T-matrix estimated with use of Eq. (17) is scalar: $T(\varepsilon) \approx V /[1-\operatorname{Vg}(\tilde{\varepsilon})]$, the matrix structure of next GE terms is essentially defined by the structure of interaction matrix:

$$
\hat{A}_{\mathbf{n}}=T\left(g_{\mathbf{n}}+\hat{\tau}_{y} \mathrm{e}^{i \hat{\tau}_{z} \theta_{\mathbf{n}}} f_{\mathbf{n}}\right) \cos (\mathbf{K} \cdot \mathbf{n}) .
$$

Here $\mathrm{e}^{i \theta_{\mathbf{n}}}=\left(n_{x}+i n_{y}\right) / n$ and the two propagator functions are approximated at low energies, $|\tilde{\varepsilon}| \ll W$, and not too short distances, $n \gg a$, as:

$$
g_{n} \approx \frac{i \pi \tilde{\varepsilon}}{W^{2}} H_{0}^{(2)}\left(\frac{\tilde{\varepsilon} n}{\hbar v_{F}}\right), f_{n} \approx \frac{3 \pi t}{W^{2}} \frac{a}{n} .
$$

involving the zeroth order Hankel function $H_{0}^{(2)}$ [28].

Calculation of the pair GE term in Eq. (14) is simplified by the two facts:

i) since the fast oscillating factor has zero average:

$$
\overline{\cos (\mathbf{K} \cdot \mathbf{n})}=\frac{1}{N} \sum_{n} \cos (\mathbf{K} \cdot \mathbf{n})=0,
$$

the odd part of the pair term can be neglected beside the even part, 
ii) the even part is defined by the diagonal form of the matrix product:

$$
\hat{A}_{\mathbf{n}}^{j j^{\prime}}=\hat{j} \hat{A}_{\mathbf{n}} \hat{j}^{\prime} \hat{A}_{-\mathbf{n}} \hat{j} \approx \hat{j} T^{2} \frac{g_{\mathbf{n}}^{2} \delta_{j, j^{\prime}}+f_{\mathbf{n}}^{2}\left(1-\delta_{j, j^{\prime}}\right)}{2},
$$

(using the average: $\overline{\cos ^{2}(\mathbf{K} \cdot \mathbf{n})}=1 / 2$ ). The related correction to $\hat{\Sigma}$ is a scalar $\Sigma_{2} \sim c^{2} V^{3} / W^{2}$, that is small enough beside the T-matrix term $c T \approx c V$ to assure convergence of the renormalized GE. The contribution to $\Sigma_{2}$, non-vanishing at $|\tilde{\varepsilon}| \rightarrow 0$, is due to interactions between impurities of different types $\left(j \neq j^{\prime}\right)$ realized by the functions $f_{n}$ and amounts to:

$$
\Sigma_{2} \approx \frac{\pi^{2} c^{2} V^{3}}{W^{2}} \ln \sqrt{\left(\frac{W}{\pi V}\right)^{2}-1} .
$$

Note that $\operatorname{Im} \Sigma_{2}$ by Eq. (23) is non-zero only if the perturbation parameter is not too weak: $|V| \gtrsim W / \pi$ (though this restriction is easier than by the above $V_{\text {cr }}$. Otherwise, for $W / \pi \gtrsim|V| \gtrsim W / \pi^{2}$, the maximum GE correction to $\operatorname{Im} \hat{\Sigma}$ would come from impurity quadruples: $\operatorname{Im} \Sigma_{4} \sim c^{4} V^{5} / W^{4}$, and so on. Anyway, such power low (in $c$ ) contributions would dominate over the exponentially small $\rho_{s c, \min }$ in the minimum DOS under impurity effect. Also, smallness of these contributions assure the IRM criterion, Eq. (18), and thus the band-like type of states through entire low energy range.

\section{Vacancy perturbations}

The defects most commonly discussed in graphene are vacancies and the following analysis concentrates on their effects in this material. Since vacancy perturbation has no explicit energy parameter, its modeling finds a certain freedom of choice and the most usual approach in literature uses the model for vacancy by the before considered local perturbation, Eq. (11), in the limit of $V \rightarrow \infty$ [15]. Such a strong assumption may look somewhat artificial physically, so it can be of interest to check its validity by a comparison with a more realistic perturbation model. To this end, we employ here the mechanism of simple exclusion of the hopping processes from and to the vacancy sites in the Hamiltonian, Eq. (1), this perturbation being expressed in terms of the local operators as:

$$
H^{\prime}=-t\left[\sum_{\mathbf{p}_{1}, \boldsymbol{\delta}}\left(a_{\mathbf{p}_{1}}^{\dagger} b_{\mathbf{p}_{1}+\boldsymbol{\delta}}+\text { h.c. }\right)+\sum_{\mathbf{p}_{2}, \boldsymbol{\delta}}\left(b_{\mathbf{p}_{2}}^{\dagger} a_{\mathbf{p}_{2}-\boldsymbol{\delta}}+\text { h.c. }\right)\right] \text {. }
$$

Then, using Eq. (5), the perturbation is rewritten in the spinor form, analogous to Eq. (11):

$$
H^{\prime}=\frac{1}{2 N} \sum_{\mathbf{k}, \mathbf{k}^{\prime}} \psi_{\mathbf{k}}^{\dagger} \sum_{j, \mathbf{p}_{j}} \mathrm{e}^{i\left(\mathbf{k}^{\prime}-\mathbf{k}\right) \mathbf{p}_{j}} \hat{V}_{\mathbf{k}, \mathbf{k}^{\prime}}^{(j)} \psi_{\mathbf{k}^{\prime}},
$$

with the scattering matrices $\hat{V}_{\mathbf{k}, \mathbf{k}^{\prime}}^{(1)}=-t\left(\gamma_{\mathbf{k}}^{*} \hat{\tau}_{-}+\gamma_{\mathbf{k}^{\prime}} \hat{\tau}_{+}\right)$and $\hat{V}_{\mathbf{k}, \mathbf{k}^{\prime}}^{(2)}=-t\left(\gamma_{\mathbf{k}}^{*} \hat{\tau}_{+}+\gamma_{\mathbf{k}^{\prime}} \hat{t}_{-}\right)$. Also we apply below this model to study the effects of inter-vacancy interactions using the respective GE's.

Table 1. Basic elements and their multiplication rules for the self-energy problem in graphene with vacancies

\begin{tabular}{c|c|c|c|c}
\hline \hline & $\hat{\tau}_{+}$ & $\hat{\tau}_{-}$ & $\hat{\tau}_{1}$ & $\hat{\tau}_{2}$ \\
\hline \hline$\hat{\tau}_{+}$ & 0 & $\hat{\tau}_{1}$ & 0 & $\hat{\tau}_{+}$ \\
$\hat{\tau}_{-}$ & $\hat{\tau}_{2}$ & 0 & $\hat{\tau}_{-}$ & 0 \\
$\hat{\tau}_{1}$ & $\hat{\tau}_{+}$ & 0 & $\hat{\tau}_{1}$ & 0 \\
$\hat{\tau}_{2}$ & 0 & $\hat{\tau}_{-}$ & 0 & $\hat{\tau}_{2}$ \\
\hline \hline
\end{tabular}

But the important difference of the vacancy non-local perturbation, Eq. (25), from the local perturbation, Eq. (11), is the momentum $k$ dependence of the $\hat{V}$ matrices. Their treatment is facilitated by the fact that the constituent matrices $\hat{\tau}_{ \pm}$, together with the above defined $\hat{\tau}_{1,2}$, form a simple algebra with the multiplication rules given by Table 1 . Then, for instance, the fully renormalized GF matrix in this case is similar to Eq. (13):

$$
\hat{G}_{\mathbf{k}}=\left(\hat{g}_{\mathbf{k}}^{-1}-\hat{\Sigma}_{\mathbf{k}}\right)^{-1} \text {. }
$$

Here the self-energy matrix $\hat{\Sigma}_{\mathbf{k}}=\hat{\Sigma}_{\mathbf{k}}^{(1)}+\hat{\Sigma}_{\mathbf{k}}^{(2)}$ include the particular terms

$$
\hat{\Sigma}_{\mathbf{k}}^{(j)}=c_{j} \hat{T}_{\mathbf{k}}^{(j)}\left[1+c_{j} \sum_{\mathbf{n} \neq 0}\left(\mathrm{e}^{-i \mathbf{k} \cdot \mathbf{n}} \hat{A}_{\mathbf{n}}^{(j)}+\ldots\right)+\ldots\right],
$$

for $j$ th type vacancies and the interaction matrices $\hat{A}_{\mathbf{n}}^{(j)}=N^{-1} \Sigma_{\mathbf{k}}^{\prime} \mathrm{e}^{i \mathbf{k}^{\prime} \cdot \mathbf{n}} \hat{G}_{\mathbf{k}^{\prime}} \hat{T}_{\mathbf{k}^{\prime}}^{(j)}$. The formal structure of the GE pair term in Eq. (26) is analogous to that in Eq. (14) for impurities but, in contrast to that case, here the same symmetry condition $\sum_{\mathbf{k}} \gamma_{\mathbf{k}} F\left(\varepsilon_{\mathbf{k}}\right) \approx 0$ makes only effective interactions to be those between vacancies of the same type $\left(j=j^{\prime}\right)$.

The above T-matrices can be found as momentumdiagonal values, $\hat{T}_{\mathbf{k}}^{(j)} \equiv \hat{T}_{\mathbf{k}, \mathbf{k}}^{(j)}$, of more general forms:

$$
\hat{T}_{\mathbf{k}, \mathbf{k}^{\prime}}^{(j)}=\sum_{\mathbf{n}=0}^{\infty} N^{-\mathbf{n}} \sum_{\mathbf{k}_{1}, \ldots, \mathbf{k}_{n}} \hat{V}_{\mathbf{k}, \mathbf{k}_{1}}^{(j)} \hat{G}_{\mathbf{k}_{1}} \ldots \hat{G}_{\mathbf{k}_{n}} \hat{V}_{\mathbf{k}_{n}, \mathbf{k}^{\prime}}^{(j)}
$$

From this definition, a matrix equation is readily obtained:

$$
\hat{T}_{\mathbf{k}, \mathbf{k}^{\prime}}^{(j)}=\hat{V}_{\mathbf{k}, \mathbf{k}^{\prime}}^{(j)}+\frac{1}{N} \sum_{\mathbf{k}_{1}} \hat{V}_{\mathbf{k}, \mathbf{k}_{1}}^{(j)} \hat{G}_{\mathbf{k}_{1}} \hat{T}_{\mathbf{k}_{1}, \mathbf{k}^{\prime}}^{(j)}
$$

and, using the $\tau$-algebra by Table 1 , solved in the form:

$$
\hat{T}_{\mathbf{k}, \mathbf{k}^{\prime}}^{(j)}=\hat{V}_{\mathbf{k}, \mathbf{k}^{\prime}}^{(j)}+\frac{t^{2} \gamma_{\mathbf{k}} \gamma_{\mathbf{k}^{\prime}}^{*}}{\varepsilon} \hat{\tau}_{j}+\left(\varepsilon-\frac{1}{G(\varepsilon)}\right)\left(1-\hat{\tau}_{j}\right) .
$$

Under the natural assumption that vacancies are equally present in two sublattices: $c_{1}=c_{2}=c / 2$, and taking account of identities: $\hat{\tau}_{1}+\hat{\tau}_{2}=1$ and $\hat{\tau}_{+}+\hat{\tau}_{-}=\hat{\tau}_{x}$, the full T-matrix results as:

$$
\hat{T}_{\mathbf{k}}=-\frac{1}{G(\varepsilon)}+\frac{\varepsilon^{2}+\varepsilon_{\mathbf{k}}^{2}}{\varepsilon}-t \operatorname{Re} \gamma_{\mathbf{k}} \hat{\tau}_{x}
$$


where the first term describes a characteristic zero-energy resonance by vacancy and just presents the $V \rightarrow \infty$ limit of the above momentum-independent $\hat{T}$ for impurities while the momentum-dependent terms are non-resonant and only weakly renormalize the dispersion law at higher energies.

It is also natural to use the T-matrix, Eq. (30), "truncated" to its resonant part, in a self-consistent equation for local GF, as a $V \rightarrow \infty$ limit of Eq. (19):

$$
G_{s c}=g\left(\varepsilon+\frac{c}{G_{s c}}\right)
$$

whose solution would, in particular, tend to a finite imaginary limit: $G_{s c}(\varepsilon \rightarrow 0) \sim i \sqrt{c \ln (1 / c)} / W$. However extending of this solution towards zero energy in the case of vacancies is essentially restricted by the failure of the IRM criterion in a certain vicinity of the (here unshifted) nodal point: $|\varepsilon| \lesssim \Delta_{0}$. Approximating Eq. (30) to its first term with $G(\varepsilon) \approx G_{s c}(\varepsilon)$, such vicinity is estimated from Eq. (18) as:

$$
\Delta_{0} \sim \sqrt{\frac{c}{\ln (1 / c)}} W
$$

to define the Mott's mobility edges [21] between band-like and localized states in this disordered system. Also, the limit of band-like states and hence of the self-consistent treatment is manifested here by failing convergence of the renormalized GE. The latter is checked by comparing the pair term similar to Eq. (23), but with $V$ replaced by $G_{s c}^{-1}(\varepsilon)$, and the T-matrix term written respectively as $-c / G_{s c}(\varepsilon)$.

Otherwise, calculation of DOS within validity of the renormalized GF, Eq. (26), in the approximation of full T-matrix, Eq. (30), but taking $G(\varepsilon) \approx g(\varepsilon)$, leads to the analytic result:

$$
\begin{aligned}
\rho_{T}(\varepsilon)=- & \frac{\varepsilon}{2 \pi c W^{2}} \operatorname{Im}\left\{\left(1+\frac{1}{R_{\varepsilon}}\right) \ln \left[1-\left(\frac{2 c W}{\varepsilon+\varepsilon R_{\varepsilon}}\right)^{2}\right]+\right. \\
& \left.+\left(1-\frac{1}{R_{\varepsilon}}\right) \ln \left[1-\left(\frac{2 c W}{\varepsilon-\varepsilon R_{\varepsilon}}\right)^{2}\right]\right\}
\end{aligned}
$$

where $R_{\varepsilon}=\sqrt{1+4 c+4 c^{2} /[\varepsilon g(\varepsilon)]}$. This function within its validity is close to the "truncated" T-matrix result:

$$
\rho_{T^{\prime}}(\varepsilon)=\frac{1}{\pi} \operatorname{Im}\left[\left(\varepsilon+\frac{c}{g(\varepsilon)}\right) \ln \left[1-\left(\frac{W g(\varepsilon)}{\varepsilon g(\varepsilon)+c}\right)^{2}\right]\right]
$$

as shown in Fig. 2, and both them deviate down from the non-perturbed linear $\rho^{0}(\varepsilon)$. But at approaching the mobility edges, $|\varepsilon| \sim \Delta_{0}$, they also notably deviate from the self-consistency behavior (which should be in principle more reliable until the renormalized GE converges).
At least, within the range of localized states, $|\varepsilon| \lesssim \Delta_{0}$, the choice should be done for the non-renormalized GF, in analogy with Eq. (16). Its contribution in the simplest $\mathrm{T}$ matrix approximation: $\hat{\sigma} \approx c / g(\varepsilon)$, used in Eq. (16), leads to the expression for DOS by localized states:

$$
\rho_{\mathrm{loc}}(\varepsilon) \approx \frac{c}{\varepsilon \ln ^{2} W / \varepsilon},
$$

that intercepts the before considered $\rho_{s c}$ at such low energies. Nevertheless its validity is also limited by the GE convergence requirement, to some more narrow vicinity of zero,

$$
\Delta_{1} \sim \frac{\sqrt{c}}{\ln (1 / c)} W
$$

estimated with use of similar expressions as for the above case of impurities, Eq. (31), but replacing $G_{s c}(\varepsilon)$ by $g(\varepsilon)$, This vicinity should measure the area of concentration broadening around the vacancy resonance peak.

A closer insight on the localization process can be obtained, beginning from a single vacancy on site $p$ (say, of type 1) and constructing a quasiparticle state with energy $\varepsilon:\left|\psi_{\varepsilon}\right\rangle=\sum_{\mathbf{n}}\left(\psi_{\mathbf{n}}^{(1)} a_{\mathbf{n}}^{\dagger}+\psi_{\mathbf{n}}^{(2)} b_{\mathbf{n}}^{\dagger}\right)|0\rangle$, whose amplitudes on type $\mathrm{j}$ sites $\psi_{\mathbf{n}}^{(j)}$ are found from the Schroedinger equation, $\left(H+H^{\prime}-\varepsilon\right)\left|\psi_{\varepsilon}\right\rangle=0$. The solutions having central symmetry with respect to the impurity site $p$ are of the same form as the above referred propagator functions, Eq. (21), dominated at $\varepsilon \rightarrow 0$ by the scale-free $f_{n}$. The resulting marginal behavior of zero-energy states by single defects [15], though does not correspond to their true localization, facilitates localization of states on defect pairs (and their higher numbers). For instance, a pair of vacancies at long enough separation $r \gg a$ produces a doublet

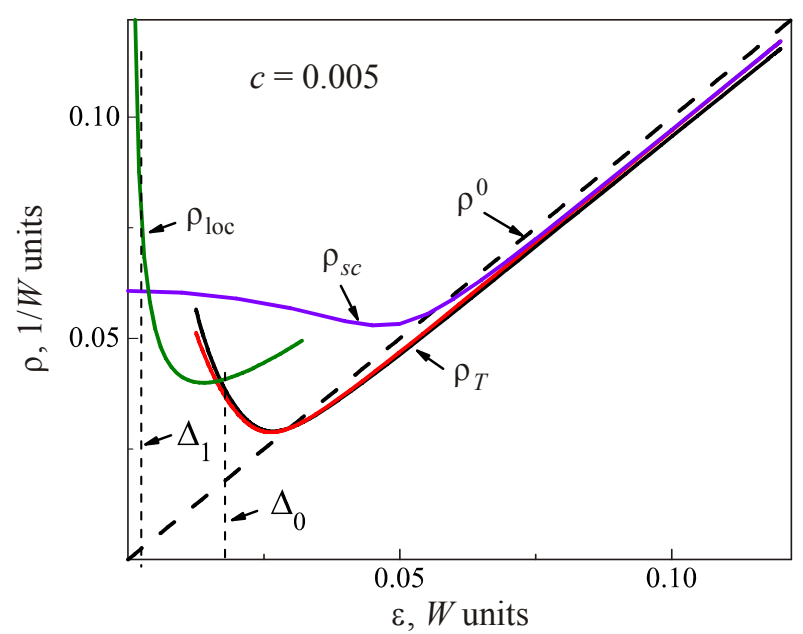

Fig. 2. Results of different approximations for DOS in graphene with vacancies at their concentration $c=0.005$ in comparison with the non-perturbed linear law $\rho^{0}(\varepsilon)$. The thin dashed lines indicate the estimated limits of validity of renormalized GE $\left(\Delta_{0}\right.$, the Mott's mobility edge) and of non-renormalized GE $\left(\Delta_{1}\right)$. 
of localized levels at finite energies $\pm \varepsilon_{r}$ where $\varepsilon_{r} \sim W a /[r \ln (r / a)]$, both having characteristic radius $r_{\text {pair }} \sim r \ln (r / a) \gg r$, thus yet much bigger than the separation between these vacancies. This situation can be seen in the spirit of general theory of of quasiparticle spectra in disordered systems [20,22], as presence of a whole series of defect pairs with separations ranging from $\sim a$ to $\sim \bar{r} \sim a / \sqrt{c}$ (the average distance between single defects, a reasonable biggest size of an isolated pair). Accordingly, the biggest pair size will define the closest distance, $\Delta_{1}$, from a pair level to zero energy, and the effectively smallest size (but not too rare in the system) will define the mobility edge $\Delta_{0}$.

Existence of a finite mobility gap $\left[-\Delta_{0}, \Delta_{0}\right]$ around zero energy should produce important practical effects on electron dynamics and transport in the disordered graphene system. Though vacancies themselves should not perturb the initial electron-hole symmetry and zero location of the Fermi energy $\varepsilon_{F}$, it can be modified by some other mechanisms like adsorbed impurities or chemical bias. Then, with its crossing the mobility edge by a related control, a transition from insulating to metallic state can occur in this system at zero temperature and a very high temperature sensitivity of transport in the vicinity of such transition can be expected.

It is of interest to compare this type of spectrum restructuring with the known examples for non-relativistic spectra. Thus, the low-frequency acoustic resonance $\omega_{\text {res }} \sim \omega_{D} \sqrt{M / M^{\prime}} \ll \omega_{D}$ by impurities with mass $M^{\prime} \gg M$ in a crystal with atomic mass $M$ and Debye frequency $\omega_{D}$ [32] gives rise to splitting of phononic spectrum near $\omega_{\text {res }}$ with opening of a quasi-gap (seen as a dip in DOS) of width $\sim c \omega_{D}^{2} / \omega_{\text {res }}$, at surpassing the characteristic impurity concentration $c_{0} \sim\left(\omega_{\text {res }} / \omega_{D}\right)^{3}$ (the cubic law) [33]. This dip corresponds to repulsion of band levels from the impurity resonance (in contrast to the hump in DOS and attraction of band levels to the resonance expected in graphene). Another example is the donor level $\varepsilon_{\text {loc }}$ near a parabolic conductance band [31], which rapidly expands and merges with this band when the donor concentration exceeds $c_{\mathrm{loc}} \sim \varepsilon_{\mathrm{lgc}} / E_{m}$ (linear law), in the 2D case, or $c_{\text {loc }} \sim\left(\varepsilon_{\text {loc }} / E_{m}\right)^{3 / 2}$, in the 3D case. The 3/2 law also defines the characteristic concentration $c_{0} \sim\left(\omega_{\text {res }} / J\right)^{3 / 2}$ of weakly coupled $\left(J^{\prime} \ll J\right)$ impurity spins in a Heisenberg ferromagnet when the magnon spectrum splits near the resonance frequency $\omega_{\text {res }} \sim J^{\prime} \ll J$, and the cubic law $c_{0} \sim\left(\omega_{\text {res }} / J\right)^{3}$ defines such effect in an aniferromagnet [22]. Hence the case of defects in graphene, where the critical concentration appears to be either impossibly high: $c_{0} \sim 1$ (for substitutional impurities), or completely vanishing: $c_{0} \rightarrow 0$ (for vacancies), differs from all those, even at seemingly identical linear dispersion (as for phonons and antiferromagnons).

\section{Conclusions}

In conclusion, the effects of local perturbation by various types of impurities, including vacancies, in single layer graphene were studied by means of Green function techniques. Namely, restructuring of electronic quasiparticle spectrum near its relativistic Dirac-type nodal points was analyzed through application of various types of group expansions for a Green function itself or for its inverse (self-energy) in groups of interacting impurity clusters. A special study for substitutional impurities with realistic (not too strong compared to the non-perturbed bandwidth) perturbation potential permitted to conclude on persistence of band-like states in the whole nodal area, though the account of non-trivial cluster terms by the group expansion provides essential corrections (power-law in impurity concentration $c$ ) to the finite density of nodal states, besides an exponentially small $\left(\sim \mathrm{e}^{-1 / c}\right)$ value by the common $\mathrm{T}$ matrix term, and should so essentially modify the related low-energy physics. Similar group expansions in the case of vacancies, indicated the conditions for quasiparticle localization near nodal points and location of the Mott's mobility edges around them for any small vacancy concentration $c$. A possibility for specific metal/insulator transition in a graphene layer at presence of vacancies, under modulation of the Fermi energy by some additional mechanisms, is indicated. The developed group expansion approach can be useful for other types of Green functions in the systems with nodal points in quasiparticle spectrum under disorder effects.

\section{Acknowledgements}

Y.P. thanks V.A. Miransky, J.P. Carbotte and S.G. Sharapov for very useful discussions of disorder effects in graphene, and kind hospitality by the Department of Applied Mathematics of University of Western Ontario during his sabbatical stay there, when this work was initiated. The support from Portuguese Fundação para a Ciência e a Tecnologia is gratefully acknowledged. V.L. thanks the SCOPES grant and also the Special Program of fundamental researches by Physics and Astronomy Department of NAS of Ukraine for the partial support.

1. K.S. Novoselov, A.K. Geim, S.V. Morozov, D. Jaing, Y. Zhang, S.V. Dubonos, I.V. Grigorieva, and A.A. Firsov, Science 306, 666 (2004).

2. P.R. Wallace, Phys. Rev. 77, 622 (1947).

3. G. Semenoff, Phys. Rev. Lett. 53, 2449 (1984).

4. K.S. Novoselov, A.K. Geim, S.V. Morozov, D. Jaing, M.I. Katsnelson, I.V. Grigorieva, S.V. Dubonos, and A.A. Firsov, Nature 438, 197 (2005)

5. Y. Zhang, Y.-W. Tan, H.L. Störmer, and P. Kim, Nature 438, 201 (2005). 
6. V.P. Gusynin and S.G. Sharapov, Phys. Rev. Lett. 95, 146801 (2005).

7. V.P. Gusynin, V.A. Miransky, and I.A. Shovkovy, Phys. Rev. Lett. 73, 3499 (1994); E.V. Gorbar, V.P. Gusynin, V.A. Miransky, and I.A. Shovkovy, Phys. Rev. B66, 045108 (2002).

8. D.V. Khveshchenko, Phys. Rev. Lett. 87, 206401 (2001); ibid. 87, 246802 (2001).

9. Y. Kopelevich, P. Esquinazi, J.H.S. Torres, and S. Moehlecke, J. Low Temp. Phys. 119, 691 (2000).

10. B.I. Shklovskii and A.L. Efros, Electronic Properties of Doped Semiconductors, Springer (1984).

11. P.A. Lee, Phys. Rev. Lett. 71, 1887 (1993).

12. W.A. Atkinson, P.J. Hirschfeld, and A.H. McDonald, Phys. Rev. Lett. 85, 3922 (2001).

13. H. Suzuura and T. Ando, Phys. Rev. Lett. 89, 266603 (2002).

14. D.V. Khveshchenko, Phys. Rev. Lett. 97, 036802 (2006).

15. N.M.R. Peres, F. Guinea, and A.H. Castro Neto, Phys. Rev. B73, 125411 (2006).

16. E.V. Savchenko, G. Zimmerer, and V.E. Bondybey, J. Lumin. 129, 1866 (2009).

17. E.V. Savchenko, A. Ogurtsov, I. Khyzhnniy, G. Stryganyuk, and G. Zimmerer, Phys. Chem. Chem. Phys. 7, 785 (2005).

18. O.N. Grygorashchenko, V.V. Rudenkov, I.V. Khyzhnniy, E.V. Savchenko, M. Frankowski, A.M. Smith-Gickorn, M.K. Beyer, and V.E. Bondybey, Fiz. Nizk. Temp. 29, 1147 (2003) [Low Temp. Phys. 29, 876 (2003)].
19. V.L. Bonch-Bruevich and S.V. Tyablikov, The Green Function Method in Statistical Mechanics, North-Holland, Amsterdam (1962).

20. I.M. Lifshitz, Sov. Phys. JETP 17, 1159 (1963).

21. N.F. Mott, $A d v$. Phys. 16, 149 (1967).

22. M.A. Ivanov, V.M. Loktev, and Yu.G. Pogorelov, Phys. Rep. 157, 209 (1987).

23. D.N. Sheng, L. Sheng, and Z.Y. Weng, cond-mat/0602190.

24. P.W. Anderson, Phys. Rev. 109, 1492 (1958).

25. R.J. Elliott, J.S. Krumhansl, and P.A. Leath, Rev. Mod. Phys. 44, 468 (1974).

26. A.F. Ioffe and A.R. Regel, Progr. Semicond. 4, 26 (1960).

27. Yu.V. Skrypnyk and V.M. Loktev, Phys. Rev. B73, 241402(R) (2006).

28. M. Abramowitz and I. Stegun, Handbook of Mathematical Functions, National Bureau of Standards (1972), p. 358.

29. V.M. Loktev and Yu.G. Pogorelov, Phys. Rev. B79, 1442 (2001).

30. H.O. Pierson, Handbook of Carbon, Graphite, Diamond, and Fullerenes: Properties, Processing, and Applications, Noyes Data, New York (1993).

31. M.A. Ivanov, V.M. Loktev, Yu.G. Pogorelov, and Yu.V. Skripnik, Sov. J. Low. Temp. Phys. 17, 377 (1991).

32. Yu.M. Kagan and Ya.A. Iosilevskii, JETP 15, 182 (1962).

33. M.A. Ivanov, Sov. Phys. Solid State 12, 1508 (1971). 